\title{
High Resolution System for Nanoparticles Hyperthermia Efficiency Evaluation
}

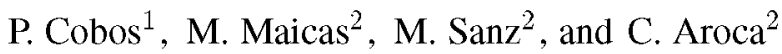 \\ ${ }^{1}$ University Institute of Automobile Research, Universidad Politécnica de Madrid (INSIA-UPM), 28031 Madrid, Spain \\ ${ }^{2}$ Institute for Systems based on Optoelectronics and Microtechnology, Universidad Politécnica de Madrid (ISOM-UPM), \\ 28040 Madrid, Spain
}

\begin{abstract}
A system to evaluate nanoparticles efficiency in hyperthermia applications is presented. The method allows a direct measurement of the power dissipated by the nanoparticles through the determination of the first harmonic component of the in quadrature magnetic moment induced by the applied field. The magnetic moment is measured by using an induction method. To avoid errors and reduce the noise signal a double in phase demodulation technique is used. To test the system viability we have measured nanowires, nanoparticles and copper samples of different volumes to prove by comparing experimental and modeled results.
\end{abstract}

Index Terms-Hyperthermia, in-phase demodulation, induced magnetic moment, magnetic nanoparticles, magnetism, nanoparticles.

\section{INTRODUCTION}

$\mathbf{N}$ OWADAYS the biomedical use of nanoparticles in diagnostic and cancer treatments is becoming a hot topic and a great effort is done to develop its medical use [1]-[4]. In cancer treatment nanoparticles are commonly used to produce hyperthermia [5], [6] and a great effort is dedicated to improve magnetic nanoparticles for their use in this application. The nanoparticles characterization for hyperthermia applications is usually done by calorimetric procedures [7]-[10]; the temperature change with time for nanoparticles dispersed in an adequate media (i.e., agar+ agar) is measured when a high frequency magnetic field is applied. This method does not allow quantitative evaluations because the measured effect, the temperature change with the time, depends on parameters such us sample holder heat dissipation or its heat capacity. On the other hand, these methods require a large amount of nanoparticles. The development of measurement methods for fast and direct nanoparticle characterization can be an interesting task. The characterization system should allow the testing of small quantities of nanoparticles in order to reduce time and cost of production. It is also desirable that these methods can perform measurements on samples with small amounts of nanoparticles in order to allow the study of the nanoparticles obtained by other flexible methods like sputtering, that produces typically few milligrams per hour [5].

In this work we propose to determine the nanoparticles efficiency to produce hyperthermia by a direct measurement of the energy dissipated during its magnetization processes. The method allows the measurement of samples with tenths of micrograms. Besides it avoids one of the most usual problems, the sample heating by Joule effect in the production coils that is one of the major error sources in the calorimetric methods.

\section{EXPERIMENTAL SYSTEM}

In this work, we propose to determine the nanoparticles efficiency to produce hyperthermia by measuring directly the energy dissipated during their magnetization processes under the action of an AC magnetic field. Assuming that the magnetic field applied to the nanoparticles, $\mathrm{H}$, is harmonic, and being Ho its amplitude, $\mathrm{T}$ the period, $\mathrm{w}$ the angular frequency, $\mathrm{H}=\mathrm{Ho}$ $\sin (w t)$, the nanoparticles magnetization, $\mathrm{M}$, can be written as a function of time as

$$
M=\sum_{k=1}^{n} a_{k} \sin (k w t)+b_{k} \cos (k w t)
$$

being

$$
\begin{aligned}
& a_{k}=\frac{1}{T} \int_{0}^{\frac{2 \pi}{w}} M(t) \cos (k w t) d t \\
& b_{k}=\frac{1}{T} \int_{0}^{\frac{2 \pi}{w}} M(t) \operatorname{sen}(k w t) d t .
\end{aligned}
$$

The density of energy dissipated in one hysteresis loop, Ws:

$$
\begin{aligned}
W s & =\oint H d M \\
& =\int_{0}^{T} H_{0} \sin (w t)\left(\sum_{k=1}^{n}\left(a_{k} w \cos (k w t)-b_{k} w \sin (k w t)\right)\right) d t \\
& =\frac{2 \pi}{2} b_{1} H_{0}
\end{aligned}
$$

the power dissipated by the nanoparticles per unity of volume is then

$$
P_{r}=\frac{W s}{T}=\frac{w}{2} b_{1} H_{0}
$$




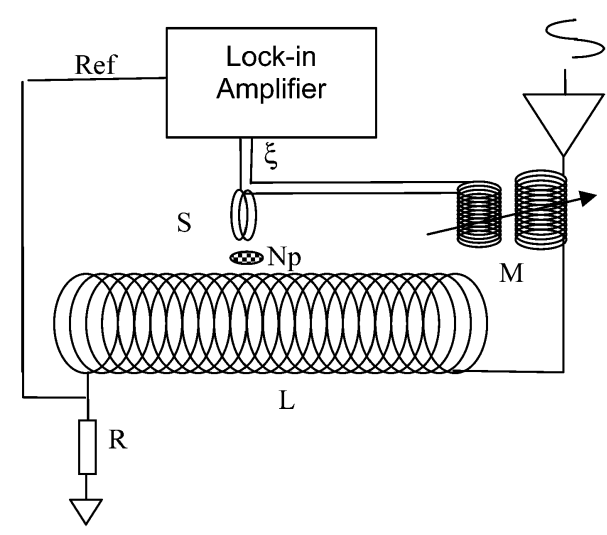

Fig. 1. Inductive method. Being: $\mathrm{L}$ the solenoid to produce the $\mathrm{H}$ field, $\mathrm{Np}$ the nanoparticles sample, $\mathrm{S}$ the secondary coil to measure the $\mathrm{Np}$ magnetization changes, $M$ the mutual inductance to compensate the $H$ flux in $S, R$ an non inductive resistance to measure $\mathrm{H}$ and to synchronize the Lock-in amplifier.

So, their efficiency to produce hyperthermia can be determined by measuring the in-quadrature first harmonic amplitude of the magnetization. The in-phase term

$$
P_{i}=\frac{W s}{T}=\frac{w}{2} a_{1} H_{0}
$$

gives us the power interchanged with the applied magnetic field. In our case, the problem is reduced to determine the power dissipation of a small volume of nanoparticles, with a total magnetic moment $\mathbf{m}(\mathbf{H})$.

To perform these measurements a simple inductive method can be used as the one schematized in Fig. 1 similar to that used in [11].

The electromotive force (EMF) induced in the secondary $\xi$ coil is produced by the changes in magnetization of the nanoparticles and by the field generated by the solenoid. This latter part is compensated with the variable mutual inductance $M$. The sources of errors in this experimental setup are:

a) phase between the field and the reference depends on the frequency because of the auto inductance of the secondary coils;

b) maximum current in the solenoid is also frequency dependant;

c) temperature of the solenoids produces a drift on the $\mathrm{H}$ compensation in $\xi$.

To solve these problems we have used a double in phase demodulation setup as shown in Fig. 2. We use two secondary coils S1 that are in series opposition to compensate the magnetic flux produced by $\mathrm{H}$. They are placed in the center of the solenoid $\mathrm{L}$, where the field $\mathrm{H}$ is almost constant. In spite of that it is very difficult to obtain a perfect $\mathrm{H}$ flux compensation because of the inherent small differences between the two coils. To solve this problem other two coils S2 are added to the system. They are in the same support than S1 coils and they are placed in the zone were the magnetic field produced by the solenoid changes abruptly. The number of turns of S2 coils (10 turns) is 20 times lower than S1 coils (200 turns) and the distance between S1 and

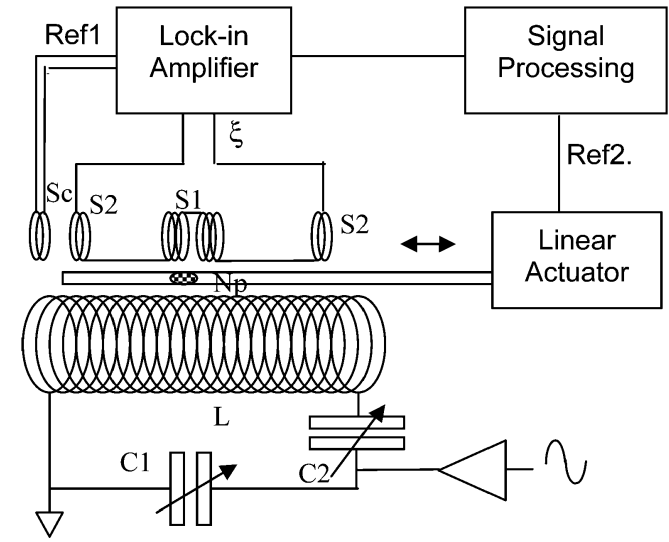

Fig. 2. Final measuring setup with a solenoid $\mathrm{L}$ to produce the $\mathrm{H}$ field, nanoparticles sample $\mathrm{Np}$, set of secondary coils connected in series opposition S1, two other secondaries coils S2 connected in series with each one of the S1coils. The nanoparticles samples $\mathrm{Np}$ moves in the region between the two $\mathrm{S} 1$ coils. Sc is a secondary coil fixed inside $\mathrm{L}$ to generate the Lock-in reference signal, Ref1.

S2 coils has been calculated for the size of $\mathrm{L}$ to obtain a linear flux change as the coil system is displaced coaxially inside $\mathrm{L}$. So, by displacing all these secondary coils system with a non metallic micro positioning, an almost perfect $\mathrm{H}$ compensation is obtained. The exciting magnetic field $\mathrm{H}$ is produced by the current flowing through the solenoid plus the magnetic field produced by the eddy currents induced in the solenoid wires. So, the secondary and all the system has been carefully designed to compensate the signals induced for both fields and for the signals produced by the sample holder. The sample, $\mathrm{Np}$, is displaced periodically by a mechanical actuator between the two main secondary coils $\mathrm{S} 1$ (the period can be varied from $1 \mathrm{~s}$ to 15 s) and 20 periods are digitized taking, at least, 60 measurements per period. By making a Fourier analysis of the measurement the maximum value of the signal induced in the secondary coils is obtained so the thermal drift and other noises are avoided. The distance between the two S1 coils has been calculated to obtain a linear response and with maximum slope of the signal induced in the $\mathrm{S} 1$ coils when $\mathrm{Np}$ is displaced from one coil to the other. The main source of errors comes from an incorrect adjust in the phase of the lock-in amplifier reference signal. To avoid this problem, the reference signal Ref1 is obtained from a very low impedance coil, Sc, placed in a fixed position inside the solenoid. The impedance of this coil, Lc, is much lower than the input resistance of the reference input of the lock-in amplifier $\mathrm{R}_{\text {ref. }}$. Considering (1), the in-phase of the first harmonic induced EMF in the secondary coils system will be proportional to $a_{1} w$ $\cos (w t)-b_{1} w \sin (w t)$, so the in-phase signal measured by the lock-in amplifier is proportional to $\mathrm{a}_{1} \mathrm{w}$ and the in-quadrature signal proportional to $b_{1} \mathrm{w}$. Another source of errors is found in the phase change due to secondary coils $\mathrm{S} 1+\mathrm{S} 2$ impedance Ls. To minimize this error the impedance has been reduced as much as possible being much lower than lock-in input impedance $R_{\text {in }}$. In any case we have

$$
\frac{L_{c}}{R_{r e f}}=\frac{L_{s}}{R_{\text {in }}}
$$




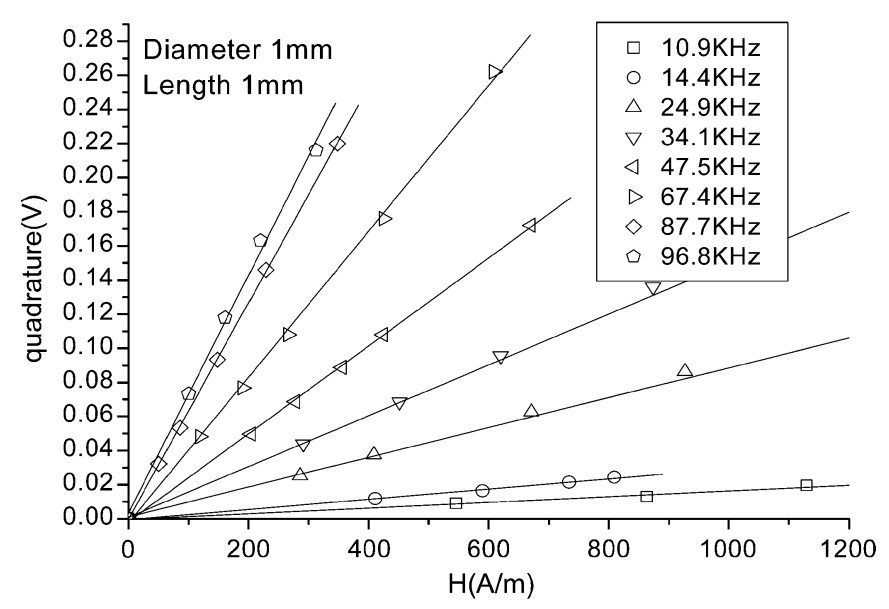

Fig. 3. In quadrature signal proportional to $b_{1} w$, vs. applied field amplitude.

The excitation current is obtained by using a quasi resonant configuration. $\mathrm{Lw}-1 / \mathrm{wC}_{2}$ is hold always positive and equal to $1 / \mathrm{wC}_{1}$. In this way, with very low current and voltage excitation high magnetic fields, $\mathbf{H}$, are obtained. The system can be calibrated by measuring a well known gold sample particle and all the measurements can be referenced to it.

\section{ReSULTS}

To test the system we have selected copper $(\mathrm{Cu})$ samples as they have magnetic moments induced as a result of the induced eddy currents. They can be measured in a similar way as magnetic samples with their permanent magnetic moments. In a single model, one nanoparticle behaves like a small loop area $\mathrm{S}$, with a resistance $\mathrm{R}$ and self-inductance associated $\mathrm{L}^{\prime}$. It can be proved that, with this model, the power dissipated by Joule effect and the power interchanged with the applied field by the eddy currents induced are

$$
W_{R}=\frac{w^{2} H o^{2} \mu_{0}^{2} S^{2} R}{R^{2}+\left(L^{\prime} w\right)^{2}}, \quad W_{L}=\frac{w^{3} H o^{2} \mu_{0}^{2} S^{2} L^{\prime}}{R^{2}+\left(L^{\prime} w\right)^{2}} .
$$

Whereas the EMF induced in secondary coils is

$$
\frac{\text { Vquad }}{H}=\frac{K}{2} \frac{w^{2} \mu_{0}^{2} S^{2} R}{R^{2}+\left(L^{\prime} w\right)^{2}}, \quad \frac{\text { Vphase }}{H}=\frac{K}{2} \frac{w^{3} \mu_{0}^{2} S^{2} L^{\prime}}{R^{2}+\left(L^{\prime} w\right)^{2}}
$$

being $\mathrm{K}$ a dimensional $\left(\mathrm{m}^{-1}\right)$ constant that depends on the secondary coil topology and its number of turns. The geometry of the samples measured was cylindrical with diameters of $2 \mathrm{~mm}$, $1 \mathrm{~mm}$ and $0.5 \mathrm{~mm}$ and lengths of $2 \mathrm{~mm}, 1 \mathrm{~mm}$ and $0.5 \mathrm{~mm}$ respectively. This kind of samples can be also analyzed by finite element model (FEM) simulations.

Fig. 3 shows the measured in-quadrature EMF versus the applied field amplitude for different frequencies. Similar curves were obtained for the three samples. As it can be seen, in the measured range, $\mathrm{V}$ versus $\mathrm{H}$, curves shows a linear behavior. In Fig. 4 it is shown the slopes of these curves $\mathrm{V} / \mathrm{H}$, which are proportional to $\mathrm{W} / \mathrm{H}^{2}$ (watt $\mathrm{m}^{2} / \mathrm{A}^{2}$ ), versus frequency.
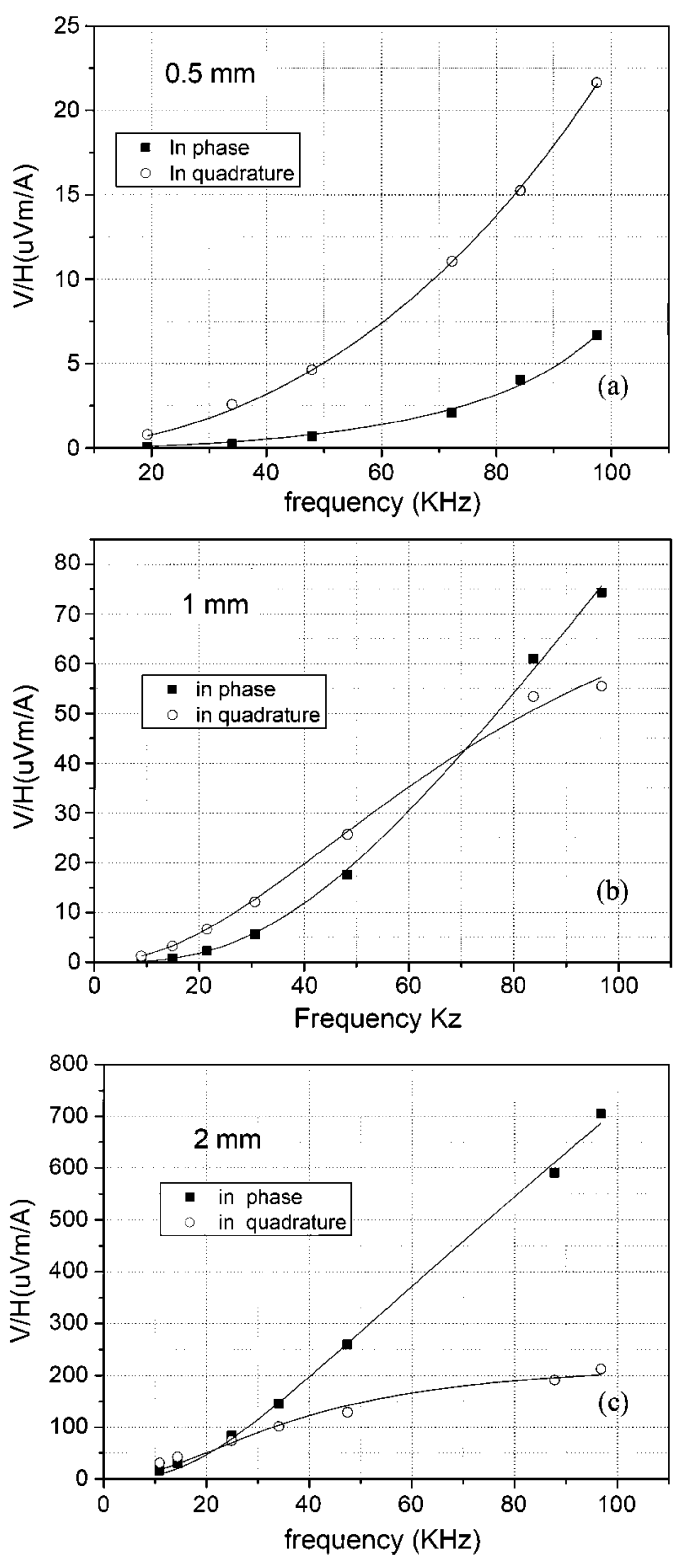

Fig. 4. Experimental results for the $\mathrm{Cu}$ samples.

The functions used to fit experimental points have been:

$$
\frac{\text { Vphase }}{H} \approx \frac{w^{2} a}{c+b w^{2}}, \quad \frac{\text { Vquad }}{H} \approx \frac{w^{3} a^{\prime}}{c+b w^{2}}
$$

which are derived from (8).

Fig. 5 show the results obtained from F.E.M. simulations to evaluate the induced currents and their relative phase with respect to the applied field. We use COMSOL ac/dc simulator to solve the Ampere Law for a harmonic solution considering the same $\mathrm{Cu}$ samples with the same geometry as those used in experimental measurements (see Fig. 4).

These curves show a magnitude proportional to the power dissipated in the sample as this is proportional as well to the induced current and the frequency. They are displayed as a function of the frequency and so they are equivalent to those in Fig. 4. 

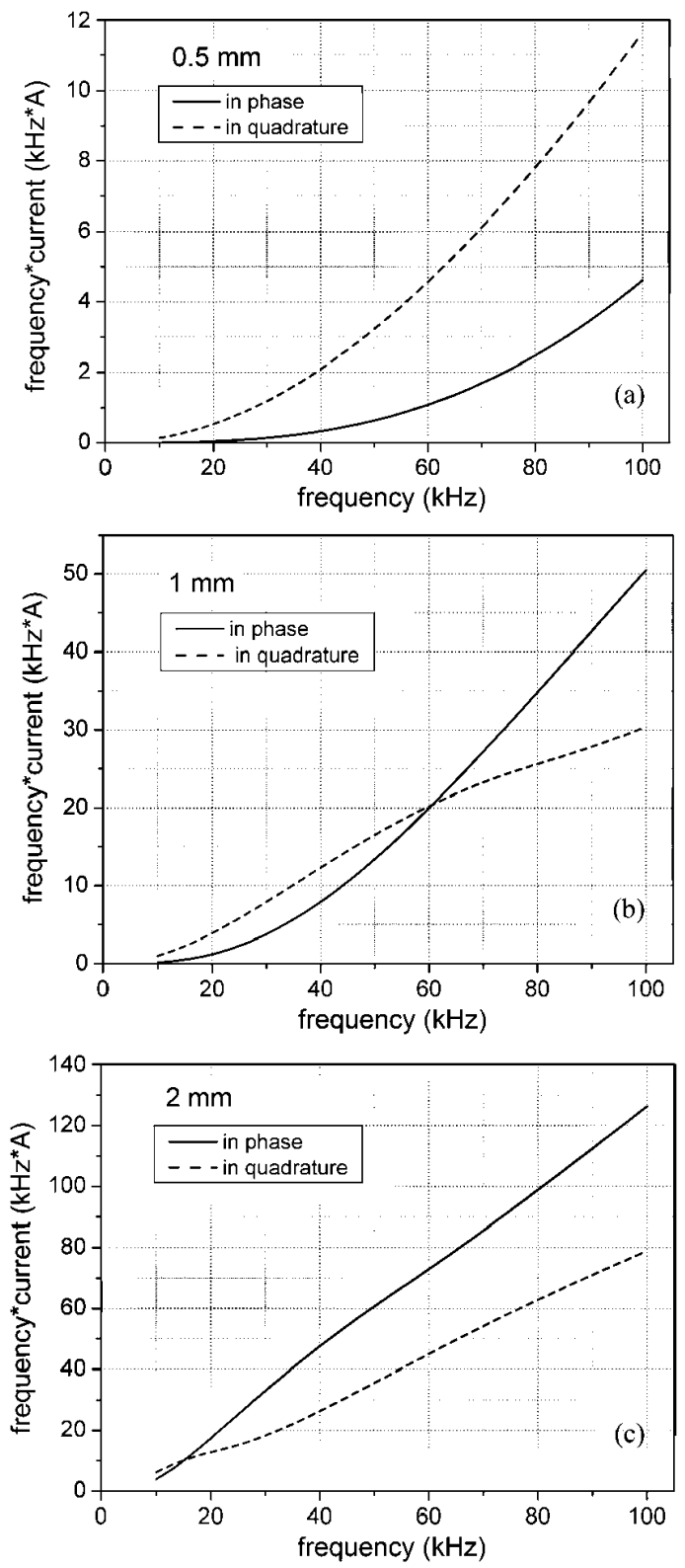

Fig. 5. Results obtained from FEM simulations for the same geometries as measured samples.

As it can be seen, there is a good agreement between the experimental measurements and the results obtained from FEM simulations. It is interesting to remark the good agreement in the intersection point between the in phase and in quadrature curves.

To prove the high sensitivity of the system we show in Fig. 6 the results from measurements on $45 \mu \mathrm{g}$ of $30 \mathrm{~nm}$ diameter $1 \mu$ length of Cobalt nanowires sample. In this case the in-quadrature power is lower than in-phase one due to their high permeability and to the small size that reduces the eddy currents to almost cero.

The main losses are due to hysteresis so the losses increase linearly with $w$. The same effect occurs for ferrite powder (spherical, $20 \mathrm{~nm} \varnothing, 5 \mathrm{mg}$ ).

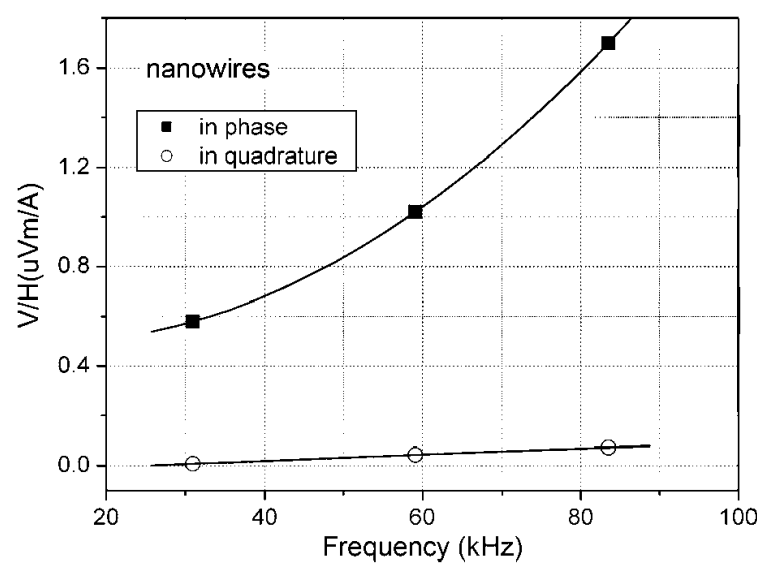

Fig. 6. Power measured on Co nanowires samples with a diameter of $30 \mathrm{~nm}$ and $1 \mu \mathrm{m}$ length.

\section{CONClusion}

We have shown that by using an inductive method it is possible to obtain information about power dissipated by induced hysteresis effects in materials with very low dimensions. The system performs these measurements using an in phase double demodulation system that allows an accurate measurement of the first harmonic in-quadrature and in-phase amplitudes. Results fit well with theoretical predictions.

\section{ACKNOWLEDGMENT}

The authors would like to thank Prof. J. M. Aroca for his discussion and L. Pérez and A. Llavona for the supply of the nanowire samples. This work was supported in part by Spanish CYCIT under Grant MAT2007-65969-C02-01 and Grant SAL$0312 / 2006$ and in part by the CAM-UPM for the fundings for Research Groups.

\section{REFERENCES}

[1] P. Hofmann-Amtenbrink, M. Hofmann. H. Montet, and X. . "Superparamagnetic nanoparticles-A tool for early diagnostics," Swiss Med. Weekly, vol. 140, pp. 7-13, Dec. 11, 2010.

[2] A. Zao, L. Tang, and J. T. Feng, "Nanothermotherapy by high performance magnetic nanoparticles," Nanomedicine, vol. 5, no. 9, pp 1305-1308, Nov. 2010.

[3] R. Frimpong and J. Hilt, "Magnetic nanoparticles in biomedicine: Synthesis, functionalization and applications," Nanomedicine, vol. 5, no. 9 , pp. 1401-1414, Nov. 2010

[4] A. Wu, A. G. Ou, and P. Zeng, "Biomedical applications of magnetic nanoparticles," Nano, vol. 5, no. 5, pp. 245-270, Oct. 2010

[5] M. Maicas, M. Sanz, H. Cui, C. Aroca, and P. Sánchez, "Magnetic properties and morphology of $\mathrm{Ni}$ nanoparticles synthesized in gas phase," J. Magn. Magn. Mater., vol. 322, pp. 3485-3489, 2010.

[6] M. Bekovic and A. Hamler, "Determination of the heating effect of magnetic fluid in alternating magnetic field," IEEE Trans. Magn., vol. 46 , no. 2, pp. 552-555, Feb. 2010.

[7] A. Pshenichnikov, V. Mekhonoshin, and A. Lebedev, "Accurate measurement of the specific absorption rate using a suitable adiabatic mag netothermal setup," Appl. Phys. Lett., vol. 92, no. 9, 2008.

[8] L. Lacroix and J. Carrey "A frequency-adjustable electromagnet for hyperthermia measurements on magnetic nanoparticles," Rev. Scientif. Instrum., vol. 79, no. 9, 2008.

[9] Y. Ma, Y. Wu, J. Zhou, Y. Sun, Y. Zhang, and N. Gu "Size dependence of specific power absorption of Fe O particles in AC magnetic field," J. Magn. Magn. Mater., vol. 268, pp. 33-39, 2004.

[10] A. Skumiel, "Suitability of water based magnetic fluid with $\mathrm{CoFe} O$ particles in hyperthermia," J. Magn. Magn. Mater., vol. 307, pp. 85-90, 2006.

[11] M. Zeisberger, S. Dutz, R. Möller, R. Hergt, N. Matoussevitch, and H. Bönnemann, "Metallic cobalt nanoparticles for heating applications," J. Magn. Magn. Mater., vol. 311, pp. 224-227, 2007. 